\title{
Foreword: special issue on particles finite element method in multi-physics problems
}

\author{
Sergio R. Idelsohn Barg1,2
}

Published online: 10 December 2016

(C) OWZ 2016

Particle-based methods in which each material particle is followed in a Lagrangian manner have been used successfully in the last years for different applications.

One of the latest evolution of particle-based methods is the Particle Finite Element Method (PFEM). The PFEM combines the particle precept with the FEM shape functions using an auxiliary FE mesh. This mesh may be quickly rebuilt at each time step (PFEM with moving mesh) or may be a fixed mesh (PFEM with fixed mesh). In the last case, the results from the Lagrangian particles are projected at each time iteration on a fixed mesh.

The idea of combining FMs with moving particles was originally used in the so-called Particle in Cell method and later in an extension called the Material Point Method (MPM), which uses an FE background mesh.

Despite that both PFEM and MPM employ a fixed FE mesh and a set of Lagrangian particles, there are important differences in the way the particles are employed; in the MPM, all computations are performed on the mesh, while in the PFEM, the aim is to calculate as much as possible on the particles, leaving small corrections to be performed on the mesh. Furthermore, the most important difference is that in PFEM, the particles do not represent a fixed amount of mass but rather material points that transport only intrinsic properties. This allows using a variable number of particles and therefore simplifying refinement.

Sergio R. Idelsohn Barg

sergio@cimne.upc.edu

1 ICREA-CIMNE, Barcelona, Spain

2 The National University of Litoral, Santa Fe, Argentina
The PFEM has been successfully used to solve the NavierStokes equations and fluid-structure interaction problems as well as solid mechanics problems. The advantages of the PFEM concerning the tracking of internal interfaces have also been explored and used to solve multi-fluid flows. The possibility to use PFEM to solve nonlinear problems with large time-steps in order to obtain an accurate and fast solution was also successfully presented for the solution of the homogeneous incompressible Navier-Stokes equations. This new strategy was named PFEM second generation (PFEM-2). The enhanced PFEM-2 version to solve multiphase problems, preserves the large time-step goodnesses of the single-phase strategy, also includes enrichment strategies to capture discontinuities in the pressure gradient, i.e., pressure kinks.

This special issue is a collection of nine technical papers presenting the state-of-the-art in modeling and simulations of solid and fluid mechanics problems at different scales involving the Particle Finite Element Method as well in his original version (PFEM) as in his new upgrade (PFEM-2).

As Editor of this Special Issue, I would like to thank all the authors for their contributions. 